\title{
"Les victimes ne veulent pas se réconcilier" : entretien avec Luisa Castro-Nilo
}

\section{Antonia GARCIA CASTRO}

\section{(2) OpenEdition}

1 Journals

\section{Édition électronique}

URL : http://journals.openedition.org/conflits/203

DOI : 10.4000/conflits.203

ISSN : $1777-5345$

Éditeur :

CCLS - Centre d'études sur les conflits lilberté et sécurité, L'Harmattan

\section{Édition imprimée}

Date de publication : 1 septembre 2000

ISBN : 2-7475-0572-3

ISSN : 1157-996X

\section{Référence électronique}

Antonia GARCIA CASTRO, «"Les victimes ne veulent pas se réconcilier" : entretien avec Luisa CastroNilo », Cultures \& Conflits [En ligne], 40 | hiver 2000, mis en ligne le 09 mars 2005, consulté le 30 mars 2021. URL : http://journals.openedition.org/conflits/203; DOI : https://doi.org/10.4000/conflits.203

Ce document a été généré automatiquement le 30 mars 2021.

Creative Commons License 


\title{
"Les victimes ne veulent pas se réconcilier" : entretien avec Luisa Castro-Nilo
}

\author{
Antonia GARCIA CASTRO
}

Pensez-vous que le nouveau régime démocratique de votre pays soit stable?

Il faut en premier lieu se demander qui sont ceux qui pourraient déstabiliser le régime, planifié et mis en place dans la seconde période de la dictature (1980-1989) via la promulgation de la Constitution de 1980, créée précisément en vue de préserver ledit régime. D'un côté, les militaires. Le seul élément qui pourrait les amener à détruire ce qu'ils ont eux-mêmes construit (ce qui ne serait pas une nouveauté, il ne faut pas oublier que le système politique instauré en 1925, via l'adoption d'une nouvelle Constitution - épisode dans lequel la participation des militaires fut un élément fondamental - a été détruit sans hésitations par d'autres militaires en 1973), c'est le fait de se sentir traqués en tant qu'institution par le thème des droits de l'homme : c'est-àdire, par les procès intentés contre Pinochet et d'autres militaires en service actif. Mais, les gouvernants de la Concertation ${ }^{1}$ ont su aborder ce thème avec doigté, en partenariat avec les militaires, de manière à éviter que le sentiment de remise en cause, ou d'offense, ne les amène à des situations extrêmes: ce qui, je crois, n'a jamais été réellement envisagé même au sein du haut commandement survivant de la dictature. Aujourd'hui, parmi les plus jeunes officiers, l'objectif est plutôt de recréer des liens avec la citoyenneté, avec les civils, tout en se fortifiant en tant qu'institution.

3 La droite civile et organisée en deux partis politiques sait par ailleurs, que pour la première fois depuis cinquante ans (le seul gouvernement de droite qu'ait connu le Chili au 20ème siècle, est celui des années 1958 et 1964) elle a la possibilité de maittriser l'Exécutif, d'être élue au gouvernement (je ne désire pas entrer dans des divagations sur " le pouvoir ", les hommes de droite l'ont toujours exercé et le seul moment où ils ont encouru le risque de le perdre - ici, le pouvoir économique - c'est durant le gouvernement de l'Unité populaire; ils ont alors utilisé leur bras armé, les Forces Armées chiliennes, ainsi que le pouvoir nord-américain). A travers les bénéfices qu'elle 
peut tirer du système électoral dit binominal ${ }^{2}$, la droite peut donner sens à l'alternance au gouvernement, ce qui fournirait au régime une occasion d'afficher sa vocation démocratique.

4 La gauche extra-parlementaire. Réduite aujourd'hui à sa plus simple expression, elle paye les " pots cassés " du socialisme réel et de ses propres erreurs. Enfermée dans un discours et une pratique qui ne parviennent pas à la rapprocher des secteurs qui ne se sentent pas représentés par le gouvernement de la Concertation. Victime du système électoral binominal, elle n'a pas réussi non plus à transformer en capital politique le mérite d'avoir lutté contre la dictature et de n'avoir par transigé avec le néolibéralisme et pas davantage avec le thème des droits de l'homme. Elle n'est pas une menace pour le régime, elle n'en a ni la force ni le désir, son objectif étant de s'y insérer et de lutter pour sa démocratisation de l'intérieur et non pas de le détruire de l'extérieur.

5 De ce point de vue, à court et moyen terme, le régime ne semble pas courir de risques. Cependant, il me semble que dans le long terme, un mécontentement profond est entrain de se développer et de générer une réaction mettant fin à l'optimisme systématique. Un grande partie de la jeunesse, mécontente, déçue, sans expectative, sans aucune voie pouvant canaliser ses inquiétudes, consciente des limitations imposées par ce régime politique, s'exclue elle-même de la participation politique traditionnelle et est fortement critique : des partis de droite, des partis du centre membres du gouvernement - et des partis de gauche qui n'ont pas su renverser la situation. Cette jeunesse avec une mentalité marginale pourrait être un facteur déstabilisateur d'un régime fondé sur l'exclusion et sur l'exploitation tout azimuts.

Quelle a été l'importance, à votre avis, de la " réconciliation " dans la transition démocratique?

7 Ici aussi, il est nécessaire de distinguer des niveaux. La réconciliation a été un thème de débat ancré dans le monde universitaire, dans le monde politique et dans le monde religieux. Elle n'est pas présente dans d'autres secteurs: les entrepreneurs font des affaires qu'ils soient réconciliés ou non; les victimes ne veulent pas se réconcilier; les disparus ne peuvent pas. Les bourreaux - quelques uns parmi eux - ceux qui courent le risque d'être condamnés à 40 ans de prison, commencent tout juste à dire qu'ils se sont peut-être trompés. La classe politique, elle, s'est réconciliée depuis longtemps et a pu faire sa Transition: il faut remonter à l'année 1985 où elle fixe les paramètres du changement. En définitive, la classe politique a décidé de recréer un pays sur la base d'un certain nombre d'arguments fallacieux: " nous avons été tous également responsables de la violence des années 1970, violence qui a conduit à la fracture institutionnelle "; " l'institution militaire n'est pas responsable des crimes d'Etat commis sous le gouvernement militaire ", etc. Le mythe fondateur du " nous sommes tous Chiliens ", une fois encore se superpose aux différences, aux haines, aux rendezvous manqués, pour créer une nation dans laquelle seulement quelques uns se reconnaissent... sauf quand il s'agit d'en appeler à un chauvinisme chevronné pour refuser à la Bolivie une partie de son territoire lui permettant d'avoir une issue à la mer ou de se protéger contre " l'invasion " d'une main d'œuvre péruvienne bon marché.

8 En synthèse, la transition (vers quoi? vers où ?) n'a eu besoin, dans la pratique, d'aucune réconciliation, si ce n'est d'un discours larmoyant fondamentalement religieux et non pas profondément politique, rationnel, susceptible de mettre en question l'essence même de la nation, de la république, susceptible aussi d'interpeller 
les mémoires, susceptible encore de générer sur la place publique, le débat que nous aimerions avoir " nous, ceux qui sommes ceux que nous sommes ", au-delà de l'isolement abrutissant auquel nous conduisent aujourd'hui Internet et la carte de crédit.

Trouvez-vous que l'on puisse justifier l'usage de l'amnistie dans les processus de " réconciliation "?

Il est évident - puisque nous parlons d'une réconciliation par le haut - qu'une amnistie par le haut paraît cohérente. L'amnistie a été décidée en 1978 alors que commençaient à apparaître des corps impudiques, massacrés et que d'autres crimes étaient connus, crimes inavouables pour les sauveurs de la patrie: il était alors nécessaire de se protéger des actions potentielles, menées par ceux qui ne comprendraient pas à quel point la lutte pour préserver la démocratie avait été dure... La transition, création magique du milieu des années 1980, a été un excellent instrument pour empêcher que les militaires ne " défilent " inutilement devant les tribunaux de justice ${ }^{3}$.

Y a-t-il des choses que vous ne pouvez pas pardonner?

12 Oui. Beaucoup. Les crimes tout d'abord. Mais non seulement ceux qui ont été commis entre 1973 et 1989. Tous les crimes qui ont jalonné notre histoire: les plus de 3000 morts des grèves du salpêtre en 1907 ; les morts de Valparaiso lors de la " semaine sanglante "; ceux de Puerto Montt en 1965 ; ceux de la mine El Salvador, en 1966. Tous ces morts qui n'ont pas mérité une enquête et pas même un débat sur la " réconciliation ". Ensuite, plus proche de nous, du point de vue des générations, nos morts, les amis, les pères, les enfants. Les torturés, les disparus, les humiliés. Mais aussi, le crime d'avoir réussi à ce que des amis trahissent, dénoncent, se dénigrent. Crime impardonnable s'il en est, enlever à l'homme sa dignité, l'obliger à choisir entre être un héros ou un traître. Les coupables n'ont pas demandé pardon, il me semble. Il n'y a pas de mot, pas de concept qui puisse permettre la rédemption de l'être humain qui ayant eu la possibilité d'agir librement a décidé de ne pas être miséricordieux.

13 Il faut encore se demander quelles sont les différentes acceptions que peut prendre le concept de pardon ou le verbe pardonner. En espagnol nous avons entre autres synonymes : absoudre, gracier, lever, exempter, comprendre, rabaisser, tolérer, libérer, " passer au-dessus de "... Passer au-dessus... De quoi ? Du fait que des crimes ont été commis. Or, même si l'humanité semble s'être habituée, et même si d'autres pays ont peut-être connu pire, ces crimes ne sont pas des quantités négligeables. " Tolérer "... quoi? Que l'être humain de manière cyclique ressente le besoin de se jeter sur ses voisins pour les mettre en pièces au nom d'on ne sait quels idéaux? " Comprendre ", quoi ? Peut-être que les hommes ne sont que cela et que l'histoire ne peut s'écrire que de cette manière et que l'on n'a pas encore appris une autre façon de régler les différences.

Pourrait-on, devrait-on, oublier les événements historiques les plus tragiques?

Si c'est de décrets dont nous parlons, alors oui, bien sûr. L'histoire comme construction se prête à cela : à oublier ce qui fâche, à garder seulement ce qui est nécessaire pour fonder cet idéal de nation que d'aucuns ont décidé de soutenir. "Pourrait-on " ou " devrait-on ", ces formulations sont ambiguës voire contradictoires. S'il s'agit de pouvoir, on peut toujours, c'est ce qu'on dit au Chili [de poderse, se puede]. De fait, l'histoire de l'humanité est construite sur des oublis volontaires des moments les plus tragiques du passé : il a fallu quelquefois des incantations magiques, telles que " nous 
avons tous été résistants "... ou telles que " nous sommes tous Chiliens " pour que tout semble avoir été oublié... Je dirais que ce qui est novateur à notre époque, c'est plutôt qu'un secteur de l'humanité ait décidé de ne pas oublier et parfois, de ne pas pardonner.

Le processus est souvent long, vingt-cinq, cinquante ans... mais une discussion intéressante est en cours aujourd'hui et elle vise les mythes fondateurs. Pour ce secteur, on ne " devrait " pas oublier. Dans le cas chilien, cela veut dire que l'on ne doit pas oublier une histoire éclaboussée de crimes, peut-être moins qu'ailleurs, peut-être...

En définitive, notre réconciliation passe, d'abord (réconciliation dans le sens que lui donne Hannah Arendt: se réconcilier avec l'idée que finalement dans l'histoire de l'humanité de telles choses arrivent) par notre capacité à accepter que nous n'avons pas réussi à construire une histoire différente. Que cette idée que nous avions dans les années 1960, 1970, de ce qu'était la spécificité chilienne - et même si nous étions conscients du fait qu'il y avait des squelettes dans les armoires... nous avions l'habitude de nous sentir fiers... - était erronée. Que non, le Chili n'était pas si démocratique, ni si civilisé... Que non, nos soldats n'étaient pas le peuple armé, qu'ils ont été l'ennemi de ce peuple qu'ils ont tenté de massacrer et que s'ils n'ont pas tout à fait réussi c'est que selon certains de leurs représentants - des " erreurs stratégiques " ont été commises. Que nos voisins nous ont quelquefois dénoncés et que dans le meilleur des cas, ils n'ont pas vu que tout près de chez eux, il y avait des centres de torture. Et enfin, que la réconciliation, à supposer qu'elle soit possible, passe nécessairement par la reconnaissance de ces crimes, par le non-oubli, par l'acceptation du fait que nos institutions se sont prêtées à ces crimes atroces, attentant contre l'idée même d'humanité. Alors, et seulement alors, il s'agira de voir quel type de nation on peut construire. C'est de cette manière qu'il est nécessaire de conjuguer les verbes pouvoir et devoir. On ne doit pas oublier pour pouvoir reconstruire la nation.

\section{NOTES}

1. NDT. La Concertation est une coalition de partis, regroupant pour l'essentiel le Parti Démocrate-Chrétien (PDC), le Parti Socialiste (PS), le Parti Pour la Démocratie (PPD). Née du programme dit d'Alliance Démocratique, en 1985, cette coalition devient " Concertation des partis politiques pour le non " lors du plébiscite du 5 octobre 1988, puis " Concertation des partis politiques pour la démocratie " lors des élections présidentielles du 14 décembre 1989, qui voit la victoire de son candidat, le démocratechrétien, Patricio Aylwin. Depuis cette date, tous les Présidents de la République ont été des représentants de la Concertation (Aylwin, PDC, 1990-1994, Frei, PDC, 1994-1999, Lagos, PS, 1999-...).

2. NDT. Le scrutin binominal, adopté lors des premières élections législatives de 1990, établit que pour obtenir deux sièges au Parlement, il faut que la liste de la plus forte votation double les votes de la liste arrivée en seconde position, si tel n'est pas le cas, ces deux listes auront le même nombre de sièges. Ce système tend à favoriser la liste 
arrivée en deuxième position, le plus souvent celle des partis de droite (Rénovation Nationale et Union Démocratique Indépendante).

3. NDT. Référence à l'argumentaire développé par le haut commandement des Forces Armées depuis 1993 : au mois de mai 1993, les commandants en chef se sont mobilisés pour témoigner publiquement leur mécontentement face à la multiplication des enquêtes pour violations des droits de l'homme impliquant des militaires en service actif et tout particulièrement le fait que lesdits militaires étaient sans cesse convoqués à comparaître devant les tribunaux de justice.

\section{INDEX}

Index géographique : Amérique du Sud, Chili

Mots-clés : victimes et victimisation, sortie de conflits

\section{AUTEUR}

\section{ANTONIA GARCIA CASTRO}

Entretien réalisé et traduit par Antonia Garcia Castro. NDT. Le mode de présentation choisi par la personne interviewée a été respecté. La présentation qui suit est la sienne. Luisa Castro Nilo est historienne, professeur à l'université ARCIS, Santiago. Née en 1950. Militante des Jeunesses Communistes à partir de 1964 et du Parti Communiste à partir de 1973. Participe de manière active et enthousiaste au projet le plus osé de l'histoire de notre pays : le gouvernement populaire (1970-1973) tente, au sein d'un système profondément injuste et discriminant, d'ouvrir un chemin de démocratisation effective. Lors du coup d'Etat (11 septembre 1973) elle est étudiante en dernière année de " Pédagogie en Histoire " et enseigne l'histoire dans le secondaire. Sous la dictature, elle participe au travail de reconstruction du Parti Communiste et milite de manière clandestine jusqu'en 1982, année où elle doit quitter le pays et s'exile. 